\title{
Editorial
}

\section{Cooperative Localization in Wireless Ad Hoc and Sensor Networks}

\author{
Davide Dardari, ${ }^{1}$ Chia-Chin Chong, ${ }^{2}$ Damien B. Jourdan, ${ }^{3}$ and Lorenzo Mucchi ${ }^{4}$ \\ ${ }^{1}$ Wireless Communication Laboratory, Department of Electronics, Computer Sciences and Systems, \\ University of Bologna, 40126 Bologna, Italy \\ ${ }^{2}$ Do CoMo Communications Laboratories USA, Inc., Palo Alto, CA 94303, USA \\ ${ }^{3}$ Rockwell Collins, Warrenton, VA 20187, USA \\ ${ }^{4}$ Department of Electronics and Telecommunications, University of Florence, 50139 Florence, Italy
}

Correspondence should be addressed to Davide Dardari, ddardari@ieee.org

Received 19 June 2008; Accepted 19 June 2008

Copyright (c) 2008 Davide Dardari et al. This is an open access article distributed under the Creative Commons Attribution License, which permits unrestricted use, distribution, and reproduction in any medium, provided the original work is properly cited.

The need for highly accurate position information is of great importance in many commercial, public safety, and military applications. With the integration of GPS into cell phones, in conjunction with WiFi localization, we are entering a new era of ubiquitous location-awareness. In the coming years, we will see the emergence of high-definition location-awareness applications: localization systems that operate in the harsh communication environments where GPS does not operate, such as inside buildings and in caves, still providing submeter localization accuracy which is not currently feasible with GPS.

Reliable localization in such conditions is a key enabler for a wide variety of applications including logistics, security tracking (the localization of authorized persons in highsecurity areas), medical applications (the monitoring of patients), search and rescue (communications with fire fighters or natural disaster victims), control of home appliances, automotive safety, and military systems, as well as in the large set of emerging wireless sensor network (WSN) applications. Other nonconventional applications of location information include networking protocols that take advantage of position information to improve the performance of routing algorithms (georouting), as well as enabling interference avoidance techniques in future cognitive radios.

One of the major requirements for most applications based on wireless ad hoc and sensor networks is accurate node localization even in the absence of infrastructure (anchor nodes). In fact, sensed data without position information is often less useful. Due to several factors (e.g., cost, size, power) only a small fraction of nodes obtain the position information of the anchor nodes. In this case, a node has to estimate its position without a direct interaction with anchor nodes; and a cooperation between nodes is needed in a multihop fashion. In some applications none of the nodes is aware of its absolute position (anchor-free) and only relative coordinates are estimated instead.

Whether the localization techniques are based on signal strength or on signal time-of-flight, measurement errors are unavoidable, especially in cluttered environments. Robust estimation signal processing schemes as well as cooperative localization algorithms are useful to improve localization accuracy.

The goal of this special issue is to bring together contributions from signal processing, communications, and related communities, with particular focus on fundamental limitations, signal processing, and new algorithm design methodologies of cooperative localization systems.

The understanding of fundamental localization performance limits in cooperative networks in the presence of unreliable measurements is of great importance since the knowledge of such limits can also help the design and comparison of new localization algorithms. This topic is covered in the first paper "Cooperative localization bounds for indoor ultra-wideband wireless sensor networks" by $\mathrm{N}$. Alsindi and K. Pahlavan where, based on empirical models of ultra-wideband width (UWB) time-of-arrival- (TOA-) based outdoor-to-indoor and indoor-to-indoor ranging, it provides cooperative localization bounds for WSNs in different indoor multipath environments: residential, manufacturing floor, old office, and modern office buildings. 
WSNs usually have to deal with limited energy and hardware resources. The paper "A wireless sensor network for RF-based indoor localization" by V. A. Kaseva et al. proposes a novel MAC protocol for location tracking using low complexity nodes where energy-efficiency and scalability are a primary concern. Experimental results on commercial devices validate the solution proposed.

The paper "Error control in distributed node selflocalization" by J. Liu and Y. Zang characterizes the effect of error propagation in distributed localization algorithms. Further, a suitable mechanism to mitigate such errors is proposed and applied to existing localization algorithms.

In the paper "A new time-based algorithm for positioning mobile terminals in wireless networks" by I. Martin-Escalona and F. Barcelo-Arroyo, a hybrid localization technique is proposed. The positioning algorithm, named time of arrival to time difference of arrival (TOAD), computes TDOA measurements from the messages that TOA-base ranging in sight stations exchange while their positioning processes are running. This study addresses the accuracy of the TOAD algorithm in two different environments: line-of-sight (LOS) and non-line-of-sight (NLOS). This technique improves the scalability and integrity of TOA techniques, making possible for the stations to position themselves without injecting network traffic.

UWB is a promising technology for accurate localization in harsh environments and it is specifically addressed in the following two papers.

The first paper "Two-step time of arrival estimation for pulse-based ultra-wideband systems" by S. Gezici et al. treats the problem of detection and TOA estimation of the first path using UWB signal. In order to accomplish both accurate TOA estimation and to reduce the estimation time, a twostep algorithm is proposed where first a coarse TOA estimate is obtained starting from received signal energy samples, then the arrival time of the first signal path is refined by considering a hypothesis testing approach.

In "The effect of cooperation on localization systems using UWB experimental data" by D. Dardari et al., an extensive measurement campaign is described and the measured data are used to derive statistical ranging models based on TOA estimation in the presence of LOS and NLOS propagation conditions. In addition, an iterative cooperative localization algorithm is proposed. Starting from measured data, the effect of the cooperation between target nodes is investigated.

The last two papers address the problem of localizing and tracking objects using a WSN. In particular, the paper "Localization capability of cooperative anti-intruder radar systems" by E. Paolini et al. considers the problem of localizing passive objects with a multistatic radar using impulse radio UWB technology. The proposed system consists of a cooperative network of one transmitting UWB node and at least three receiving nodes. The analysis begins by considering a single pair of one transmitter and one receiver. Given the transmission power and the ability of the receiver to resolve the UWB signal, the region where the target can be detected is obtained, along with the associated localization error. The impact of node location, transmitted power, and localization uncertainty are then discussed for the complete multistatic system. Given these conflicting factors, a criterion is suggested for effectively placing the transmitter and receiver nodes.

Finally, the last paper "Tracking objects with networked scattered directional sensors" by K. H. Plarre and P. R. Kumar proposes a three-phase optimization strategy for tracking multiple objects using a network of directional sensors. The objects to be tracked are assumed to be moving in straight lines and at constant speed as they cross the region. The sensors envisioned in this application have a very narrow field of view, such as lasers or highly directional temperature sensors. As the object crosses a sensor's line of sight, only the time of the detection is recorder; neither range nor angle measurements are necessary. The task of the sensors is then to estimate the directions and speeds of the objects and the sensor lines, which are unknown a priori. This estimation problem involves the minimization of a highly nonconvex cost function, and is solved using the proposed adaptive basis algorithm.

\section{ACKNOWLEDGMENTS}

Several researchers worldwide have contributed to this special issue submitting their latest research. The authors would like to thank all the reviewers for their great effort in suggesting improvements during successive iterations. They would like also to send their special thanks to the publisher, its staff, and the Editor-in-Chief for their patience and useful suggestions during the finalization of this issue. They hope that this special issue will represent a useful starting point and stimulus for further research on wireless localization technologies in the coming years.
Davide Dardari
Chia-Chin Chong
Damien B. Jourdan
Lorenzo Mucchi 